\title{
Current results of the NOvA experiment
}

\author{
Liudmila Kolupaeva ${ }^{1,2, \star}$ on behalf of the NOvA Collaboration \\ ${ }^{1}$ Joint Institute for Nuclear Research \\ ${ }^{2}$ Moscow State University
}

\begin{abstract}
NOvA is a long baseline neutrino oscillation experiment at Fermi National Accelerator Laboratory, USA. It is one of the so-called "off-beam-axis" experiments with two highly active detectors separated by $810 \mathrm{~km}$ of Earth's crust. Both detectors are identical and consist of PVC cells filled with a liquid scintillator. The NOvA collaboration has already published its first results for muon neutrino disappearance [1] and electron neutrino appearance [2] mode with data collected between July 2013 and March 2015. This talk is devoted to a discussion on NOvA's first results.
\end{abstract}

\section{Introduction}

NOvA (NuMI Off-Axis $v_{e}$-Appearance) is a long baseline neutrino oscillation experiment consisting of two functionally identical detectors, which have been placed $14 \mathrm{mrad}$ off-axis. The NuMI (Neutrinos at the Main Injector) facility at the Fermi National Accelerator Laboratory (USA) provides a muon (anti-)neutrino beam for the experiment. The neutrino beam, with a peak energy of $2 \mathrm{GeV}$, travels $810 \mathrm{~km}$ through the Earth's crust to the Far Detector at Ash River (Minnesota). Muon neutrinos may oscillate to electron or tau neutrinos as they propagate. The NOvA detectors can measure the transition probabilities $P\left(v_{\mu} \rightarrow v_{e}\right)$ and $P\left(v_{\mu} \rightarrow v_{\mu}\right)$. From these measurements NOvA is able to extract oscillation parameters: $\Delta m_{32}^{2}$, mixing angle $\theta_{23}$, CP violating phase $\delta_{C P}$ and neutrino mass hierarchy.

NOvA published its first results [1], [2] this year. These results are based on data collected between July 2013 and March 2015. More data and results will be presented at the Neutrino 2016 summer conference.

\section{The NOvA experiment}

Neutrinos are created by colliding $120 \mathrm{GeV}$ protons with a carbon target. The $\pi$ and $\mathrm{K}$ mesons produced will decay into a neutrino and an associated lepton. The $4.2 \mathrm{~m} \times 4.2 \mathrm{~m} \times 15 \mathrm{~m}$ near detector is located at a distance of about $1 \mathrm{~km}$ from the target and has mass about $300 \mathrm{t}$. It is used for measuring the neutrino flux composition before oscillations. The far detector is located at a distance $810 \mathrm{~km}$. It has dimensions of $15.6 \mathrm{~m} \times 15.6 \mathrm{~m} \times 60 \mathrm{~m}$ with a mass of $14 \mathrm{kt}$. It is responsible for measuring neutrino oscillations. Both detectors are placed 14 mrad off of the neutrino beam axis. Such a scheme helps to obtain narrow energy spectra near the first oscillation maximum and suppresses the high energy tail.

^e-mail: ldkolupaeva@yandex.ru 
The NOvA detectors are huge tracking calorimeters with excellent segmentation. They consist of many PVC cells $(6 \mathrm{~cm} \times 4 \mathrm{~cm})$ filled with mineral oil liquid scintillator with $5 \%$ admixture of pseudocumene. These cells are constructed in alternate planes, thus NOvA can measure the vertical and horizontal position of an event. The number of cells in the far and near detector are 344054 and 21192 respectively. Each cell contains a wavelength-shifting fiber loop with 32-pixel avalanche photodiods used for readout.

The NOvA detectors were under construction during the first analysis data taking period, from July 2013 to March 2015. During this period $3.45 \times 10^{20}$ POT were delivered to the carbon target. Because the NOvA Far detector size was not constant during this period the NOvA data should be matched to $2.74 \times 10^{20}$ POT-14-ton-equivalent.

\section{The $v_{\mu}$ Disappearance Results}

The $v_{\mu}$ Disappearance mode is sensitive to $\theta_{23}$ and $\Delta m_{23}^{2}$ measurements. During the first analysis period NOvA detected $33 v_{\mu}$ CC events instead of the $211.8 \pm 12.5$ events expected under non-oscillation hypothesis. It is a clear observation of muon neutrino oscillations. The oscillation parameter extraction procedure is based on measured spectra in both detectors. Spectra from the Near Detector (Figure 1 ) is extrapolated to the Far detector and this prediction is compared with far detector measurements. The extrapolation procedure is discribed in Figure 2. Figure 3 shows the final measured far detector neutrino spectra.

Expected background events for the NOvA disappearance analysis are 2 neutral current and 1.4 cosmic ray events. Main systematic contributions are due to Calorimetric Energy Calibration, Muon Energy Scale etc. Total systematic uncertainty for $\sin ^{2} \theta_{23}$ is $6.8 \%$ and $\Delta m_{32}^{2}$ is $3.7 \%$.

The best fit oscillation parameters are:

$$
\begin{gathered}
\Delta m_{23}^{2}=2.52_{-0.18}^{+0.20} \times 10^{-3} \mathrm{eV}^{2} \quad 0.38<\sin ^{2} \theta_{23}<0.65(68 \% \mathrm{CL}) \quad \text { (for normal hierarchy) } \\
\Delta m_{23}^{2}=-2.56 \pm 0.19 \times 10^{-3} \mathrm{eV}^{2} \quad 0.37<\sin ^{2} \theta_{23}<0.64(68 \% \mathrm{CL}) \quad \text { (for inverted hierarchy) }
\end{gathered}
$$

Allowed regions for oscillation parameters in comparison with other experiments measurement are shown in the Figure 4.

\section{The $v_{e}$ Appearance Results}

The $v_{e}$ appearance channel is sensitive to the mass hierarchy and $\delta_{C P}$ phase. The NOvA collaboration developed two selection algorithms for the $v_{e}$ analysis. The official analysis LID (Likelihood Identification) is based on $\mathrm{dE} / \mathrm{dx}$ likelihood calculations independently for transverse and longitudinal slices under different particle hypotheses. Another algorithm is LEM (Library Event Matching) [7]. Information about energy deposition is compared to the huge library of Monte Carlo events.

The extrapolation procedure is also used for far detector prediction (Figure 5 shows near detector spectra). The expected number of background events and their composition is shown in table 1 . The systematic uncertainty for the $v_{e}$ analysis is $17.6 \%$. The main contribution to systematics comes from calibration, interaction uncertainties and scintillator saturation.

NOvA observed 6 events selected by LID and 11 events selected by the LEM algorithm (Figure 6). Figure 7 shows the allowed regions for $\sin ^{2} 2 \theta_{13}$ for all values of $\delta_{C P}$ and both hierarchies. For fixed $\sin ^{2} 2 \theta_{13}=0.086 \pm 0.005$ [5], [6] figure 8 shows the significance at witch different hypotheses are disfavored for all values of $\delta_{C P}$ and both hierarchies, with fixed $\sin ^{2} \theta_{23}=0.5$.

LID analysis disfavors the $0<\delta_{C P}<0.8 \pi$ range for inverted hierarchy at $90 \%$ C.L. LEM analysis disfavors inverted hierarchy for all values of $\delta_{C P}$ and $0<\delta_{C P}<\pi$ for normal hierarchy at $90 \%$ C.L. 


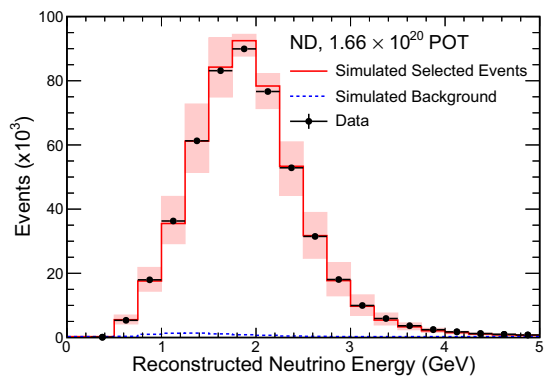

Figure 1. Near Detector spectra, which is used in extrapolation and prediction spectra in Far Detector.

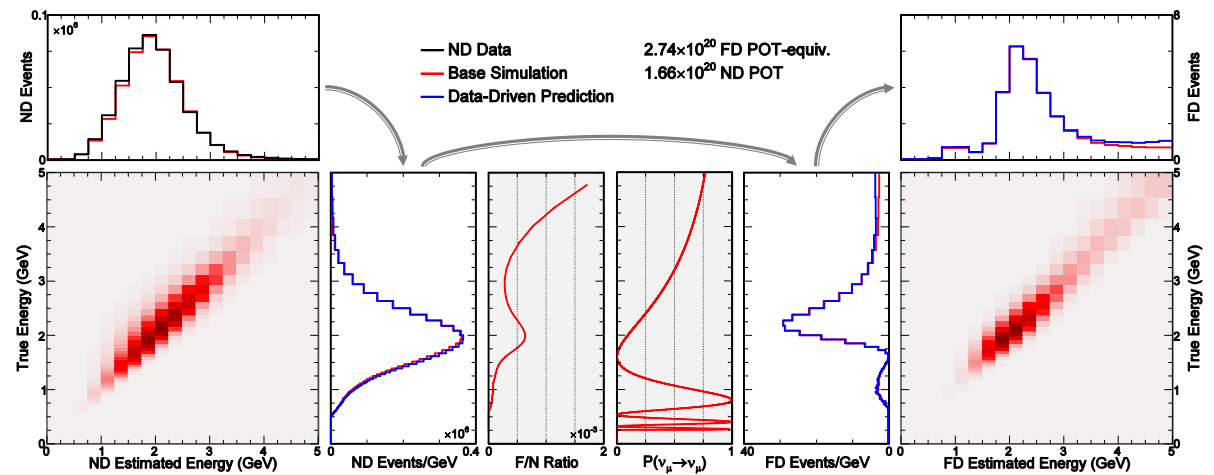

Figure 2. Extrapolation procedure with measured near detector spectra. Measured near detector spectra converted to true energy is multiplied by far-to-near detector ratio and oscillation probability. Calculated far detector true energy spectra are converted to reconstructed energy spectrum.

\section{References}

[1] P. Adamson et al. [NOvA Collaboration], Phys. Rev. D 93, no. 5, 051104 (2016)

[2] P. Adamson et al. [NOvA Collaboration], Phys. Rev. Lett. 116, no. 15, 151806 (2016)

[3] P. Adamason et al., Phys. Rev. Lett. 112, 191801 (2014)

[4] K. Abe et al., Phys. Rev. D 91, 072010 (2015)

[5] F. P. An et al., Phys. Rev. Lett. 115, 111802 (2015)

[6] Y. Abe et al., JHEP 141086 (2014)

[7] C. Backhouse and R. B. Patterson, Nucl. Inst. Meth. A 778 (2015) 

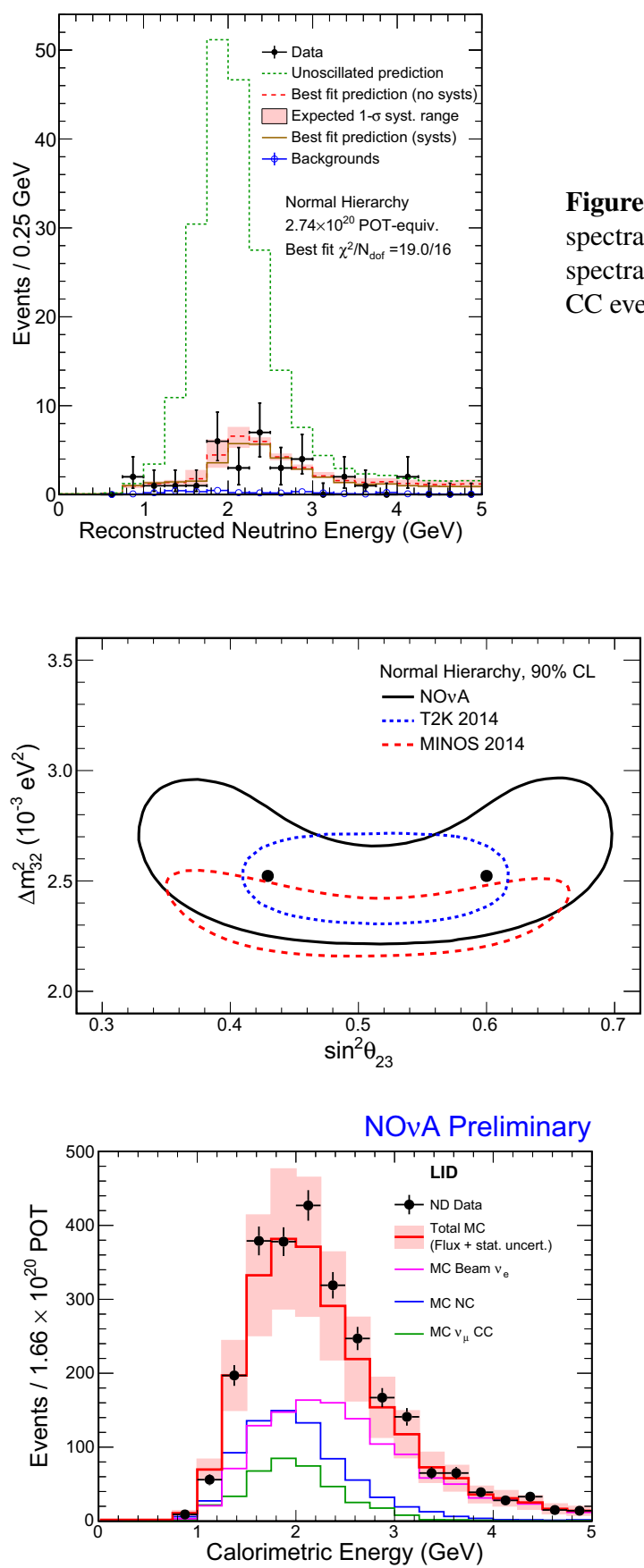

Figure 3. This figure shows the Far Detector $v_{\mu}$ energy spectra. The green line represents the non-oscillated energy spectra with an expected 211.8 events. NOvA observed $33 v_{\mu}$ $\mathrm{CC}$ events.
Figure 4. $90 \%$ C.L. contour for normal hierarchy on the surface $\sin ^{2} \theta_{23}$ and $\Delta m_{32}^{2}$ along with MINOS [3] and T2K [4] results. This plot shows good agreement.
Figure 5. Near Detector LID spectra used for $v_{e}$ analysis prediction in Far Detector. 


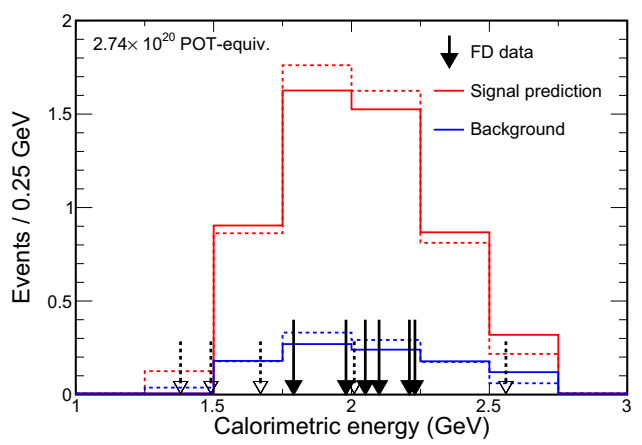

Figure 6. This figure shows both LID and LEM far detector $v_{e}$ CC energy spectrum. LID selector (solid) identified 6 events, LEM found 11 events, all LID events were selected with additional 5 events (dashed).
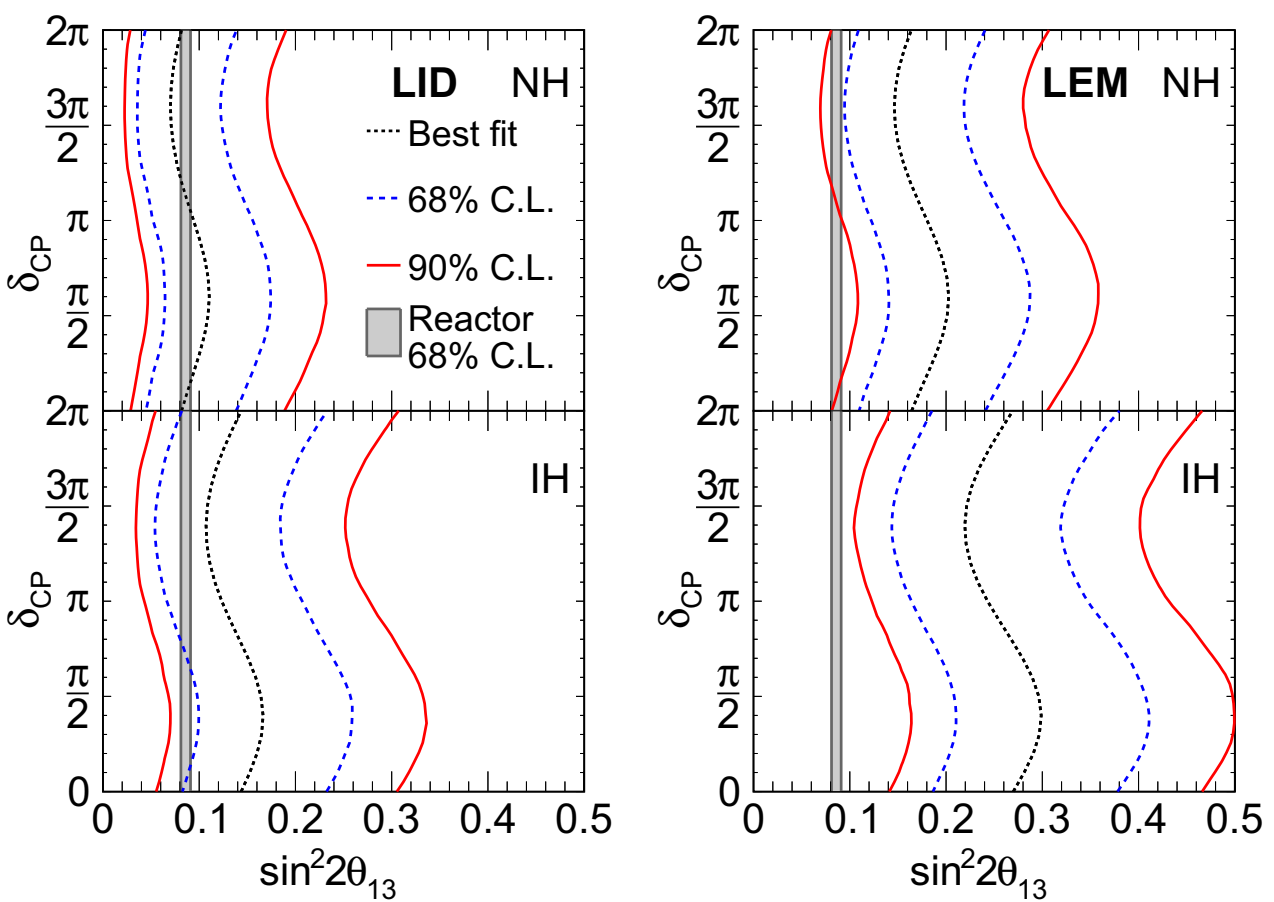

Figure 7. 68\% and 90\% C.L. regions for LID and LEM selectors in $\sin ^{2} 2 \theta_{13}$ and $\delta_{C P}$ surface in comparison with rector neutrino experiment data. The top panel is normal and the bottom is inverted hierarchy. $\sin ^{2} \theta_{23}=0.5$ remain fixed. 


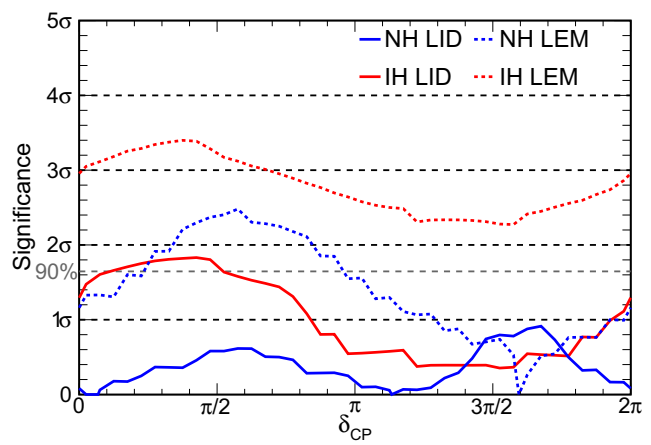

Figure 8. Significance for normal (blue) and inverted (red) neutrino mass hierarchy for all values of $\delta_{C P}$. Both LEM and LID selectors are represented.

Table 1. Expected background for $v_{e}$ analysis

\begin{tabular}{c|c|c|c|c|c|c}
\hline & Total bkg. & Beam $v_{e}$ & $\mathrm{NC}$ & $v_{\mu} \mathrm{CC}$ & $v_{\tau} \mathrm{CC}$ & Cosmic \\
\hline LID & 0.99 & 0.5 & 0.37 & 0.05 & 0.02 & 0.06 \\
LEM & 1.07 & 0.5 & 0.43 & 0.07 & 0.02 & 0.06 \\
\hline
\end{tabular}

